\title{
Boundary element modeling of horizontal grounding electrodes using the set of generalized telegrapher's equations
}

\author{
D. Poljak ${ }^{1}$, K. El Khamlici Drissi ${ }^{2}$ \& R. Goic ${ }^{1}$ \\ ${ }^{1}$ University of Split, Croatia \\ ${ }^{2}$ Blaise Pascal University, France
}

\begin{abstract}
The analysis of a horizontal grounding electrode has been carried out using the set of generalized telegrapher's equations. The integro-differential relationships arising from the full wave model are numerically handled via the GalerkinBubnov scheme of the Indirect Boundary Element Method (GB-IBEM). Some illustrative numerical results for the current and voltage induced along the horizontal electrode are given in the paper.
\end{abstract}

Keywords: boundary elements, grounding systems, antenna theory, generalized telegrapher's equations.

\section{Introduction}

Studies of grounding systems, because they are an important component in lightning protection systems (LPS), are of great interest in electromagnetic compatibility (EMC) and high voltage (HV) applications. One of the applications of particular interest nowadays is LPS for wind turbines. As they are environmentally attractive (no pollution) and wind is free to use these wind turbines have been widely used. On the other hand, they are extremely vulnerable to lightning strikes due to their special shape and isolated locations mainly in high altitude areas. Analysis of grounding systems can be undertaken by the aid of the transmission line model (TLM) [1-3] or the full wave model, also referred to as the antenna model (AM) [4-6]. The latter is considered to be the rigorous one. The key-parameter in the full wave model is the equivalent current distribution along the grounding electrode. The full wave model of the 
electrode presented in this work is based on the set of Telegrapher's equations. In particular, the effect of a lossy ground is taken into account via the corresponding reflection coefficient thus avoiding the rigorous approach based on the analytically demanding and numerically time consuming Sommerfeld integrals. The integro-differetial expressions arising from the proposed full wave model are numerically treated by means of the Galerkin-Bubnov scheme of the Boundary Element Method (GB-IBEM) [7]. Solving the set of generalized telegrapher's equations first the current distribution and subsequently the scattered voltage along the electrode are obtained.

\section{Equivalent antenna model of the grounding electrode}

The geometry of interest, shown in Fig 1, is the horizontal grounding electrode of length $L$ and radius $a$, buried in a lossy medium at depth $d$ and excited by an equivalent current source.

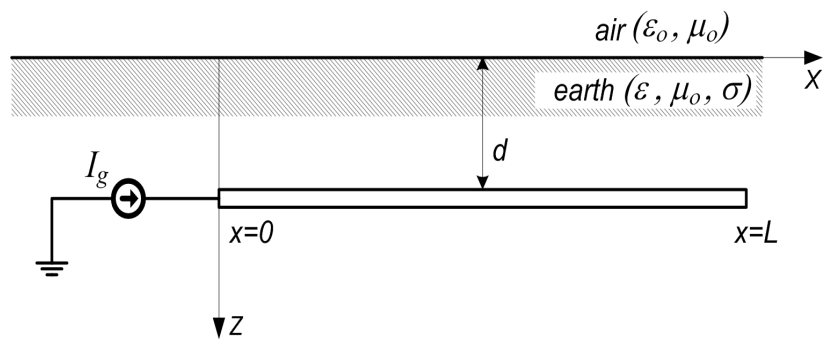

Figure 1: Horizontal grounding wire energized by a current generator $I_{g}$.

The wire dimensions are assumed to satisfy the thin wire approximation (TWA) conditions $[7,8]$.

\subsection{The generalized telegrapher's equations for a horizontal grounding electrode}

The governing equations for the current and voltage induced along the grounding electrode can be derived by enforcing the continuity conditions for the tangential components of the electric field along the wire surface.

The total field composed from the excitation field $\vec{E}^{\text {exc }}$ and scattered field $\vec{E}^{s c t}$ is equal to the product of the current along the electrode $I(x)$ and surface internal impedance $Z_{S}(x)$ per unit length of the electrode:

$$
\vec{e}_{x} \cdot\left(\vec{E}^{e x c}+\vec{E}^{s c t}\right)=Z_{s}(x) I(x) \text { on the wire surface }
$$

The surface internal impedance $Z_{s}(x)$ is given by $[9,10]$ :

$$
Z_{s}(x)=\frac{Z_{c w}}{2 \pi a} \frac{I_{0}\left(\gamma_{w a}\right)}{I_{1}\left(\gamma_{w a}\right)}
$$


where $I_{0}\left(\gamma_{w}\right)$ and $I_{1}\left(\gamma_{w}\right)$ are modified Bessel functions of the zero and first order respectively, while $Z_{c w}$ and $\gamma_{w}$ are given by $[9,10]$ :

$$
\begin{gathered}
Z_{c w}=\sqrt{\frac{j \omega \mu_{w}}{\sigma_{w}+j \omega \varepsilon_{w}}} \\
\gamma_{w}=\sqrt{j \omega \mu\left(\sigma_{w}+j \omega \varepsilon_{w}\right)}
\end{gathered}
$$

For the case of very good conductors (conductivity of order of $10^{6} \mathrm{~S} / \mathrm{m}$ ), the surface impedance $Z_{s}(x)$ can be neglected. The scattered electric field can be expressed in terms of the vector potential $\vec{A}$ and the scalar potential $\varphi$, and according to the thin wire approximation $[7,8]$ only the axial component of the magnetic potential differs from zero and it can be written:

$$
E_{x}^{s c t}=-j \omega A_{x}-\frac{\partial \varphi}{\partial x}
$$

The vector and scalar potential are, respectively, given by particular integrals:

$$
\begin{gathered}
A_{x}=\frac{\mu}{4 \pi} \int_{0}^{L} I\left(x^{\prime}\right) g\left(x, x^{\prime}\right) d x^{\prime} \\
\varphi(x)=\frac{1}{4 \pi \varepsilon_{\text {eff }}} \int_{0}^{L} q\left(x^{\prime}\right) g\left(x, x^{\prime}\right) d x^{\prime}
\end{gathered}
$$

The complex permittivity of the lossy ground $\varepsilon_{\text {eff }}$ is given by:

$$
\varepsilon_{e f f}=\varepsilon_{r} \varepsilon_{0}-j \frac{\sigma}{\omega}
$$

with $\varepsilon_{r g}$ and $\sigma$ being the corresponding permittivity and conductivity, respectively, of the ground, while $q(x)$ denotes the charge distribution along the line, $I\left(x^{\prime}\right)$ is the induced current along the line, and $g\left(x, x^{\prime}\right)$ is the corresponding Green's function of the form:

$$
g\left(x, x^{\prime}\right)=g_{0}\left(x, x^{\prime}\right)-\Gamma_{r e f} g_{i}\left(x, x^{\prime}\right)
$$

where $g_{0}\left(x, x^{\circ}\right)$ denotes the lossy medium Green function:

$$
g_{0}\left(x, x^{\prime}\right)=\frac{e^{-\gamma R_{1}}}{R_{1}}
$$

and $g_{i}\left(x, x^{\prime}\right)$ is, according to the image theory, given by:

$$
g_{i}\left(x, x^{\prime}\right)=\frac{e^{-\gamma R_{2}}}{R_{2}}
$$

while the propagation constant of the lower medium is defined as:

$$
\gamma=\sqrt{j \omega \mu \sigma-\omega^{2} \mu \varepsilon}
$$


and $R_{1}$ and $R_{2}$ are given by:

$$
R_{1}=\sqrt{\left(x-x^{\prime}\right)^{2}+a^{2}}, \quad R_{2}=\sqrt{\left(x-x^{\prime}\right)^{2}+4 d^{2}}
$$

The influence of a ground-air interface is taken into account via the following reflection coefficient (RC) [6]:

$$
\Gamma_{r e f}=\frac{\frac{1}{n} \cos \theta-\sqrt{\frac{1}{n}-\sin ^{2} \theta}}{\frac{1}{n} \cos \theta+\sqrt{\frac{1}{n}-\sin ^{2} \theta}} ; \theta=\operatorname{arctg} \frac{\left|x-x^{\prime}\right|}{2 d} ; \underline{n}=\frac{\varepsilon_{e f f}}{\varepsilon_{0}}
$$

The main advantage of RC approach versus rigorous Sommerfeld integral approach is a simplicity of the formulation and appreciably less computational cost. Generally, RC approach produces results roughly within $10 \%$ of these obtained via rigorous Sommerfeld integral approach [7]. The linear charge density and the current distribution along the electrode are related through the continuity equation:

$$
q=-\frac{1}{j \omega} \frac{d I}{d x}
$$

Substituting continuity equation (15) into (7) gives:

$$
\varphi(x)=-\frac{1}{j 4 \pi \omega \varepsilon_{e f f}} \int_{0}^{L} \frac{\partial I\left(x^{\prime}\right)}{\partial x^{\prime}} g\left(x, x^{\prime}\right) d x^{\prime}
$$

while substituting equations (6) and (16) into (5) leads to the following integral relation for the scattered electric field:

$$
E_{x}^{s c t}=-j \omega \frac{\mu}{4 \pi} \int_{0}^{L} I\left(x^{\prime}\right) g\left(x, x^{\prime}\right) d x^{\prime}+\frac{1}{j 4 \pi \omega \varepsilon_{e f f}} \frac{\partial}{\partial x} \int_{0}^{L} \frac{\partial I\left(x^{\prime}\right)}{\partial x^{\prime}} g\left(x, x^{\prime}\right) d x^{\prime}
$$

Finally, for the case of grounding electrodes the excitation function does not exist in the form of the tangential field, i.e. [4]:

$$
E_{x}^{e x c}=0
$$

Combining equations (1), (17) and (18) results in the following homogeneous integral equation of the Pocklington type for the unknown current distribution induced along the horizontal grounding electrode:

$$
j \omega \frac{\mu}{4 \pi} \int_{0}^{L} I\left(x^{\prime}\right) g\left(x, x^{\prime}\right) d x^{\prime}-\frac{1}{j 4 \pi \omega \varepsilon_{\text {eff }}} \frac{\partial}{\partial x} \int_{0}^{L} \frac{\partial I\left(x^{\prime}\right)}{\partial x^{\prime}} g\left(x, x^{\prime}\right) d x^{\prime}+Z_{s}(x) I(x)=0
$$

The knowledge of the current distribution induced along the electrode provides the assessment of the scattered voltage. To derive the telegrapher's type equations for the current and voltage induced along the electrode, the scattered voltage concept has to be included in the formulation. The scattered voltage along the horizontal electrode is defined by a line integral of a scattered vertical field component from the remote soil to the wire surface: 


$$
V^{s c t}(x)=-\int_{\infty}^{d} E_{z}^{s c t}(x, z) d z
$$

The vertical field component can be expressed in terms of the scalar potential gradient, i.e.:

$$
E_{z}^{s c t}=-\frac{\partial \varphi}{\partial z}
$$

and the scattered voltage along the wire can be written as follows:

$$
V^{s c t}(x)=\int_{-\infty}^{d} \frac{\partial \varphi}{\partial z} d z=\frac{d}{d z} \int_{-\infty}^{d} \varphi(x, z) d z
$$

Integration from the infinite soil to the conductor surface and assuming the scalar potential in the remote soil to be zero [11] and according to equation (16) leads to the scattered voltage along the electrode:

$$
V^{s c t}(x)=-\frac{1}{j 4 \pi \omega \varepsilon_{\text {eff }}} \int_{0}^{L} \frac{\partial I\left(x^{\prime}\right)}{\partial x^{\prime}} g\left(x, x^{\prime}\right) d x^{\prime}
$$

Combining equations (19) and (23) results in the set of the generalized telegrapher's equations for the horizontal grounding electrode:

$$
\begin{gathered}
\int_{0}^{L} \frac{\partial I\left(x^{\prime}\right)}{\partial x^{\prime}} g\left(x, x^{\prime}\right) d x^{\prime}+Y^{\prime} V^{s c t}(x)=0 \\
\frac{d V^{s c t}(x)}{d x}+j \omega L^{\prime} \int_{0}^{L} I\left(x^{\prime}\right) g\left(x, x^{\prime}\right) d x^{\prime}+Z_{s}(x) I(x)=0
\end{gathered}
$$

where the corresponding equivalent inductance, admittance, capacitance and conductance per unit length of the wire are given by:

$$
\begin{gathered}
L^{\prime}=\frac{\mu}{4 \pi} \\
Y^{\prime}=j 4 \pi \omega \varepsilon_{e f f}=G^{\prime}+j \omega C^{\prime}, \quad C^{\prime}=4 \pi \varepsilon, \quad G^{\prime}=4 \pi \sigma
\end{gathered}
$$

\subsection{The current source excitation}

The grounding electrode is energized by the injection of an equivalent ideal current source with one terminal connected to the grounding electrode and the other one grounded at infinity, as shown in Fig 1. This current source is included into the integro-differential equation formulation through the following boundary conditions:

$$
I(0)=I_{g}, \quad I(L)=0
$$

where $I_{g}$ denotes the impressed unit current generator. 


\section{Boundary element procedures}

The unknown current $I^{e}(x)$ along the wire segment can expressed, as follows:

$$
I^{e}\left(x^{\prime}\right)=\{f\}^{T}\{I\}
$$

Collecting the contributions from each element the integro-differential equation (19) is transferred into the following matrix equation:

$$
\sum_{j=1}^{M}[Z]_{j i}\{I\}_{i}=0, \quad \text { and } \quad j=1,2, \ldots, M
$$

where $M$ is the total number of segments and $[\mathrm{Z}]_{\mathrm{ji}}$ is the mutual impedance matrix representing the interaction of the $i$-th source with the $j$-th observation segment, respectively:

$$
\begin{aligned}
& {[Z]_{j i}=-\frac{1}{4 j \pi \omega \varepsilon_{e f f}}\left(\int_{\Delta l_{j}}\{D\}_{j} \int_{\Delta l_{i}}\left\{D^{\prime}\right\}_{i}^{T} g\left(x, x^{\prime}\right) d x^{\prime} d x+\right.} \\
& \left.+k^{2} \int_{\Delta l_{j}}\{f\}_{j} \int_{\Delta l_{i}}\{f\}_{i}^{T} g\left(x, x^{\prime}\right) d x^{\prime} d x\right)+\int_{\Delta l_{j}} Z_{L}(x)\{f\}_{j}\{f\}_{i}^{T} d x
\end{aligned}
$$

Matrices $\{f\}$ and $\left\{f^{\prime}\right\}$ contain the shape functions while $\{D\}$ and $\left\{D^{\prime}\right\}$ contain their derivatives, and $\Delta l_{i}, \Delta l_{j}$ are the widths of $i$-th and $j$-th boundary elements. A linear approximation over a boundary element is used in this work:

$$
f_{i}=\frac{x_{i+1}-x^{\prime}}{\Delta x} \quad f_{i+1}=\frac{x^{\prime}-x_{i}}{\Delta x}
$$

as this choice was proved to be optimal one in modeling various wire structures.

The excitation function in the form of the current source $I_{g}$ is taken into account as a forced boundary condition at the first node of the solution vector, i.e.:

$$
I_{1}=I_{g} \quad \text { and } \quad I_{g}=1 e^{j 0}
$$

Once the current distribution is obtained the scattered voltage defined by equation (23) can be readily evaluated using the boundary element formalism.

As the differentiation of the current distribution variation along the segment is simply given by:

$$
\frac{\partial I\left(x^{\prime}\right)}{\partial x^{\prime}}=\frac{I_{i+1}-I_{i}}{\Delta x}
$$

the scattered voltage can be computed from the following formula:

$$
V^{s c t}(x)=-\frac{1}{j 4 \pi \omega \varepsilon_{e f f}} \sum_{i=1}^{M} \frac{I_{i+1}-I_{i}}{\Delta x} \int_{x_{i}}^{x_{i+1}} g\left(x, x^{\prime}\right) d x^{\prime}
$$




\section{Numerical results}

Figure 2 shows the frequency response at the center of the electrode characterized by the following parameters: $L=20 \mathrm{~m}, d=1 \mathrm{~m}, a=5 \mathrm{~mm}$ and $I_{g}=1 \mathrm{~A}$. The ground conductivity is $\sigma=0.01 \mathrm{~S} / \mathrm{m}$ while the permittivity is $\varepsilon_{\mathrm{r}}=10$. The results computed via the BEM and the Modified Transmission Line Model (MTLM) are compared to the results obtained via NEC using Sommerfeld integral approach to account for the presence of the ground-air interface. The agreement between the results obtained via the different approaches is found to be relatively satisfactory for the given set of parameters.

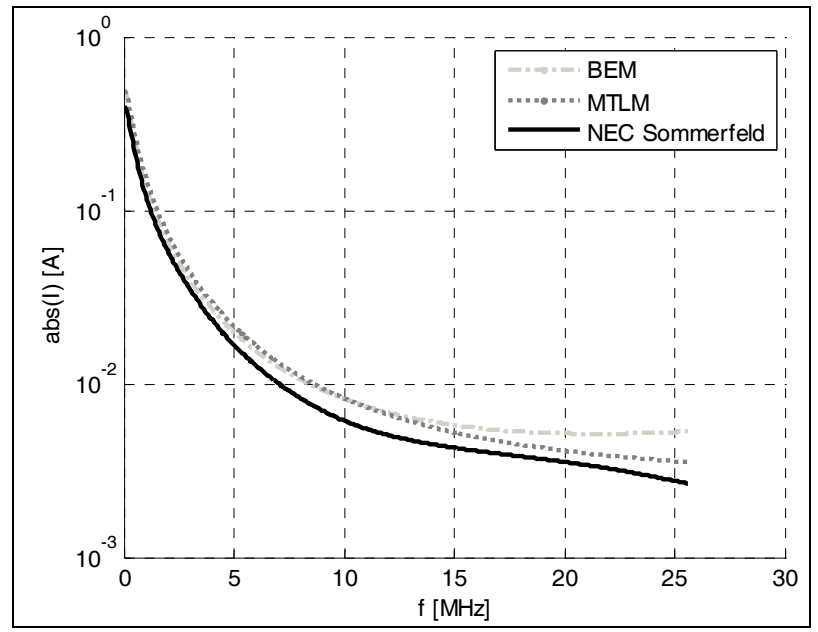

Figure 2: Current induced at the center of the grounding wire versus frequency $\left(L=20 \mathrm{~m}, d=1 \mathrm{~m}, a=5 \mathrm{~mm}, \sigma=0.01 \mathrm{~S} / \mathrm{m}, \varepsilon_{\mathrm{r}}=10\right)$.

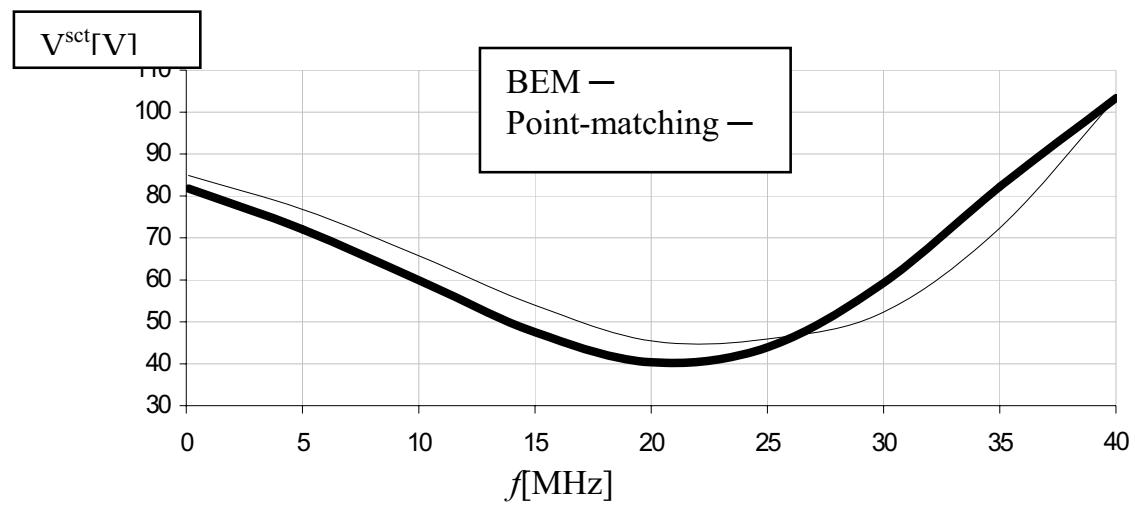

Figure 3: Voltage spectrum at the input of the grounding electrode $(L=10 \mathrm{~m}$, $\left.d=1 \mathrm{~m}, a=5 \mathrm{~mm}, \sigma=0.01 \mathrm{~S} / \mathrm{m}, \varepsilon_{\mathrm{r}}=10\right)$. 
Figure 3 shows the voltage spectrum at the driving point for a horizontal grounding electrode characterized by the following parameters: $L=10 \mathrm{~m}, d=1 \mathrm{~m}$, $a=5 \mathrm{~mm}$ and $I_{g}=1 \mathrm{~A}$. The ground conductivity is $\sigma=0.01 \mathrm{~S} / \mathrm{m}$, while the permittivity is $\varepsilon_{\mathrm{r}}=10$.

The agreement between the results obtained via BEM is in a plausible agreement with the results obtained via point matching technique.

\section{Concluding remarks}

The paper deals with modeling of grounding systems based on the generalized telegrapher's equations and related boundary element procedures. The integral relationships arising from the full wave model are numerically treated by using the Galerkin-Bubnov scheme of the Indirect Boundary Element Method (GBIBEM). The results obtained for the current distribution and scattered voltage induced along the horizontal grounding electrode agree satisfactorily with the results obtained via other methods.

Future work will involve the treatment of complex grounding systems including interconnected conductors.

\section{References}

[1] Y. Liu, M. Zitnik, R. Thottappillil, “An Improved Transmission Line Model of Grounding System”, IEEE Trans. EMC, Vol.43, No.3, pp. 348-355, 2001.

[2] G. Ala, M. L. Di Silvestre, "A Simulation Model for Electromagnetic Transients in Lightning Protection Systems", IEEE Trans. EMC, Vol.44, No.4, pp.539-534, 2003.

[3] M.I. Lorentzou, N.D. Hatziargyriou, C.Papadias, "Time Domain Analyisis of Grounding Electrodes Impulse Response”, IEEE Trans. Power Delivery, No 2., pp. 517-524, Apr. 2003.

[4] L. Grcev , F. Dawalibi, "An Electromagnetic Model for Transients in Grounding Systems”, IEEE Trans. Power Delivery, No 4., pp. 1773-1781, Oct. 1990.

[5] L. D. Grcev, F.E. Menter, "Transient Electro-magnetic Fields Near Large Earthing Systems”, IEEE Trans. Magnetics, Vol. 32, pp. 1525-1528, May 1996.

[6] D. Poljak, V. Roje, "The Integral equation method for ground wire impedance", Constanda, C., Saranen, J., Seikkala, S. (Ed), Integral methods in science and engineering, Vol. I, Longman, UK., 139-143, 1997.

[7] D. Poljak, "Electromagnetic Modelling of Wire Antenna Structures", WIT Press, Southampton-Boston, 2002.

[8] R.G. Olsen, M.C. Willis, "A Comparison of Exact and Quasi-static Methods for Evaluating Grounding Systems at High Frequencies", IEEE Trans. Power Delivery, Vol. 11, No 2, pp. 1071-1081, April 1996.

[9] F. Tesche, M. Ianoz, F. Carlsson, EMC Analysis Methods and Computational Models, John Wiley and Sons, New York 1997. 
[10] D. Poljak, F. Rachidi, S. Tkachenko, Generalized Form of Telegrapher's Equations for the Electromagnetic Field Coupling to Finite Length Lines above a Lossy Ground, IEEE Trans. EMC, Vol. 49, No 3., pp. 689-697, Aug. 2007.

[11] D. Poljak, Generalized Form of Telegrapher's Equations for the Electromagnetic Field Coupling to Buried Wires of Finite Length, IEEE Trans. EMC, 2009.

[12] J. Wallart, K. E. K. Drissi, F. Paladian, "Study of propagation constant for a single buried wire in a lossy ground," in Proc. EMC '98 Symposium, vol. 2, Roma, 1998, pp. 557-562. 